\title{
Rosalind Watkiss Singleton. “¿Crimen? No, no fue realmente un crimen": Utilizar historia oral y las memorias para enseñar historia del crimen
}

\section{Rosalind Watkiss Singleton}

Traductor. Ignacio Ayala Cordero

\section{(2) OpenEdition \\ Journals}

\section{Edición electrónica}

URL: http://journals.openedition.org/rhj/631

DOI: $10.4000 /$ rhj.631

ISSN: 0719-4153

\section{Editor}

ACTO Editores Ltda

\section{Referencia electrónica}

Rosalind Watkiss Singleton, «Rosalind Watkiss Singleton. "¿Crimen? No, no fue realmente un crimen": Utilizar historia oral y las memorias para enseñar historia del crimen », Revista Historia y Justicia [En línea], 6 | 2016, Publicado el 30 abril 2016, consultado el 19 abril 2019. URL : http://

journals.openedition.org/rhj/631; DOI : 10.4000/rhj.631 


\author{
“CCRIMEN? NO, NO FUE REALMENTE UN CRIMEN”: \\ UTILIZAR HISTORIA ORAL Y LAS MEMORIAS PARA \\ ENSEÑAR HISTORIA DEL CRIMEN ${ }^{1}$
}

Rosalind WATKISS SINGLETON²

\begin{abstract}
Resumen: Este artículo sugiere que las entrevistas orales, las autobiografías y las memorias proporcionan una nueva perspectiva para la enseñanza de la historia del crimen. El testimonio oral puede ampliar las fuentes tradicionalmente utilizadas para este propósito, otorgando perspectivas originales relativas a las actitudes populares respecto de las actividades y comportamientos criminales. Mediante el uso de entrevistas, ya sean generadas por los estudiantes o tomadas de archivos existentes de historia oral, las palabras y opiniones de los perpetradores, de las víctimas y del público en general permitiría a los estudiantes e investigadores complementar evidencias existentes como una manera de mejorar tanto su comprensión crítica de los patrones de comportamiento, como los desarrollos internos de la sociedad. La motivación de los estudiantes puede incrementarse a través de un envolvimiento más cercano con documentación directa, de aquellos que han sido afectados de alguna manera por el comportamiento criminal y de los individuos que han jugado un rol activo dentro del sistema de justicia. Los estudios comparativos y la cooperación entre estudiantes de leyes, criminología, historia y ciencias sociales sólo pueden ser renovados por el intercambio de metodologías, ideas y recursos. Este artículo utiliza la evidencia de las investigaciones personales de la autora como un caso de estudio tendiente a presentar algunas de las memorias del crimen y actitudes hacia el comportamiento criminal entre las comunidades del Black Country ${ }^{3}$ en el siglo XX.
\end{abstract}

Palabras clave: Testimonio oral, enseñar historia del crimen, cooperación interdisciplinaria, ampliar las bases metodológicas, nuevas fuentes y evidencia original, estudio de caso, percepciones del crimen, robo desde el lugar de trabajo

\footnotetext{
${ }^{1} \mathrm{NdT}$ : El artículo " 'Crime? no, it wasn't really crime': Using oral history and memoirs to teach crime history" fue publicado en Law, Crime and History, vol. 1, 2014, p. 62-82, y se puede consultar en www.pbs.plymouth.ac.uk/solon/journal/vol.4\%20issue1\%202014/Watkiss-Singleton\%20paper\%20-\%20FINAL.pdf. La Revista Historia y Justicia agradece a la autora su permiso para traducir al castellano y publicar su texto. Igualmente, se deja constancia de la edición específica, para esta traducción, realizada por Aude Argouse.

${ }^{2}$ University of Wolverhampton, r.watkiss@wlv.ac.uk.

${ }^{3} \mathrm{NdT}$ : El "Black Country", corresponde al área geográfica centro-occidental de Inglaterra, ubicada al noroeste de Birmigham, incluye las regiones de Dudley, Sandwell, Walsall y Wolverhampton. Entre las ciudades y localidades que lo componen está Tipton, Sedgley y otras citadas a lo largo del artículo. Se caracteriza por ser una región de tradición industrial.
} 


\section{Introducción}

Como las circunstancias han cambiado en la sociedad británica desde los comienzos del siglo XX, también lo han hecho las actitudes y opiniones respecto del pequeño delito y la actividad criminal tanto entre la comunidad como en el lugar de trabajo. Desde el involucramiento en el mercado negro, pasando por dos guerras mundiales y sus secuelas, hasta una variedad de crímenes dentro del lugar de trabajo, las percepciones del público raramente han coincidido con aquellas del gobierno o del sistema judicial. El conocimiento común sobre el crimen y la actividad criminal ha dependido frecuentemente de la evidencia estadística recogida por los informes judiciales y la estadística criminal. De todos modos, los datos relativos a la economía de mercado negro, y por cierto, el gris 4 , son notoriamente difíciles de discernir y cuantificar, y a menudo dependen de los informes de casos judicializados ${ }^{5}$.

Por una variedad de razones, ciertos delitos, en particular el hurto, fueron casi inevitablemente clandestinos en su naturaleza, su descubrimiento no necesariamente resulta en su judicialización y estuvieron abiertos a diferentes interpretaciones desde los puntos de vista del perpetrador, las víctimas y las autoridades. Consecuentemente, la extrapolación de evidencia estadística produce sólo un registro parcial del comportamiento criminal y provee escasa información sobre los motivos y consecuencias de tales actos ${ }^{6}$. Morris atrajo la atención respecto del hecho que "de todas las estadísticas sociales, aquellas relativas al crimen están probablemente entre las más sujetas a dudas", confiadas como están sobre "la presentación de informes de los eventos por miembros del público" y evidencia provista por la policía. El autor también atendió al hecho de que siempre subyace cierta falta de honradez a pesar que el perpetrador sea aprehendido ${ }^{7}$.

Los problemas que han revelado el uso de evidencias estadísticas también han sido destacados por Hennessey, quien indicó que "de toda la aritmética social que los historiadores utilizan para sus análisis, los cuadros criminales son los más carentes de sustancia y menos fidedignos" ". Claramente, entonces, las actividades de la economía de mercado, sea negro o gris, no pueden ser adecuadamente medidas solamente a partir de

\footnotetext{
${ }^{4} \mathrm{NdT}$ : El término "mercado gris" es un anglicismo que se refiere a la distribución de mercancías originales por intermediarios no acreditados, a través de canales no autorizados por el productor, a precios más bajos que los ofrecidos en el mercado tradicional. El mercado gris se distinguiría del mercado negro porque los productos distribuidos no son ilegales.

5 Silcock, H., The increase in crimes of theft, 1938-1949, University Press of Liverpool, 1949, p. 4; Smithies, Edward, The black economy in England since 1914, Gill \& MacMillan Humanities Press, 1984, p. 5.

${ }^{6}$ Smithies, E., The black economy, Op. Cit., p. 5.

${ }^{7}$ Morris, Terence, Crime and criminal justice since 1945, Basil Blackwell, 1989, p. 4.

${ }^{8}$ Hennessey, Peter, Having it so good: Britain in the fifties, Allen Lane, 2006, p. 83.
} 


\section{Revista Historia y Justicia}

ISSN 0719-4153 revista.historiayjusticia.org N6 - Santiago de Chile, abril 2016, p. 274-294

documentación oficial o estadísticas criminales. De todos modos, las entrevistas orales 9 pueden proporcionar ciertas luces sobre un amplio rango de actividades ilegales, incluyendo el predominio del pequeño delito en el lugar de trabajo y las comunidades locales, y pueden ayudar a revelar actitudes populares hacia los perpetradores, así como indicar el grado de involucramiento en las redes de distribución en la Inglaterra de la postguerra. Las entrevistas proveen un contra-balance a las estadísticas y fuentes convencionales.

A pesar de que no soy una especialista en los campos de la ley o la justicia criminal, sino una historiadora oral -con especialidad en historia y política-, podría sugerir que el uso de entrevistas puede probar ser una fuente adicional de utilidad para atraer el interés de los estudiantes y proveer enfoques originales para investigaciones sobre la actividad criminal. Este artículo no tiene por objeto plantear argumentos concluyentes de la eficacia de las fuentes orales para la enseñanza de la justicia criminal, más bien sugiere que la introducción de tales testimonios puede mejorar la experiencia de aprendizaje y enseñanza. Asimismo, sugiere que el uso de la evidencia oral puede ser utilizado para superar lo que Thompson llama las "brechas y debilidades de los documentos [existentes]"10. La evidencia parcial provista por las estadísticas criminales es, como Roodhouse ha indicado recientemente, más un indicativo de los métodos policiales y procedimientos jurídicos contemporáneos que de la actividad y los patrones de comportamiento criminales. Más aún, "los criminólogos frecuentemente critican a los políticos y comentaristas sociales quienes usan las estadísticas criminales como un indicador del nivel de crimen" ${ }^{11}$; seguramente, entonces, ¿una crítica similar puede hacerse sobre el exceso de confianza en dichas estadísticas en el ambiente académico, donde existen fuentes adicionales disponibles para complementar la información existente? El estudio de caso pretende demostrar cómo el uso del tes timonio oral puede revelar que las actitudes hacia el crimen no son necesariamente bien definidas y las opiniones sobre lo que puede constituir el comportamiento criminal dependen de una variedad de factores, los cuales varían según el tipo de delito, cuándo es cometido y por quién, los motivos y justificaciones para violar la ley y las percepciones fluctuantes de la sociedad para definir las actividades "ilegales". Leer (en las transcripciones) u oír (en las grabaciones) las explicaciones y experiencias de los perpetradores, las víctimas, los testigos y la gente responsable del reforzamiento de la ley, tiene el potencial de convocar el interés de los estudiantes sobre diferentes aspectos del crimen y el sistema legal.

\footnotetext{
9 Estas entrevistas provienen de mi propia investigación, una serie de más de 90 entrevistas, conducidas por la autora entre 1996 y 2013, sobre un amplio rango de temas, las cuales han sido utilizadas en mis tesis de maestría y doctorado, para ponencias en congresos académicos y para propósitos de enseñanza; y los testimonios orales recogidos por otros discutidos más adelante.

10 Thompson, Paul, The voice of the past, Oxford University Press, 2000, p. 4.

${ }^{11}$ Roodhouse, Mark, Black market, Op. Cit., p. 33.
} 


\section{Revista Historia y Justicia}

ISSN 0719-4153 revista.historiayjusticia.org No6 - Santiago de Chile, abril 2016, p. 274-294

\section{Estudio de caso: Robo desde el lugar de trabajo}

Ha sido sostenido que la transición del siglo XIX desde el trabajo a domicilio al sistema fabril fue en parte responsable del robo en el lugar de trabajo pero, como Emsley indicó, este tipo de comportamiento puede ser hallado en contextos anteriores. Por ejemplo, en la época Tudor los trabajadores a domicilio eran propensos a ser procesados si eran descubiertos "reteniendo" productos sobrantes de su trabajo ${ }^{12}$. La retención de materiales sobrantes del proceso de producción fue percibida por muchos trabajadores como gratificación o beneficio por su empleo, consagrado por la costumbre y la tradición ${ }^{13}$. La escala de esta actividad es, inevitablemente, problemática para descifrar y la precisión en estas cuestiones es imposible de lograr; no obstante, existe evidencia para sugerir que el robo de esta naturaleza escaló a través de los siglos ${ }^{14}$ y las políticas tendientes a la vigilancia de tales delitos cambiaron en consecuencia. Evaluar tal aumento constituye un reto: "nunca podríamos ser realmente claros respecto de cuánto del incremento del registro (...) reflejó un incremento 'real', y cuánto fue producto simplemente del cambio administrativo y actitudinal"15. Sin embargo, es seguro asumir, junto con Smithies, que "el robo al empleador ha sido un problema en la industria británica desde la Primera Guerra Mundial, si es que no mucho antes"16. Las restricciones en tiempo de guerra, los problemas de importar suficientes suministros y la injusta distribución de comida -hasta llegar al racionamiento en 1917-, fomentaron a aquellos con acceso a la cadena de abastecimiento a eludir el sistema y cientos, si no miles, de ciudadanos a tomar ventaja de la obtención de provisiones a través de transacciones a pequeña escala en el mercado negro. La pobreza y el desempleo en la Inglaterra entreguerras no hizo nada para frenar tales usurpaciones en el trabajo de pequeñas cantidades de bienes susceptibles de revender desde sus empleadores, u otras fuentes ilícitas, para después ofrecerlos en bares, mercados y dentro de la comunidad obteniendo una pequeña ganancia ${ }^{17}$. Claramente, la mayoría de tales transacciones basadas en pequeños hurtos no fueron detectadas y, en consecuencia, se mantuvieron fuera de las estadísticas criminales oficiales.

Por lo tanto, la utilización de fuentes de información adicionales, testimonios orales, memorias y autobiografías pueden revelar a los investigadores enfoques originales respecto de los métodos, los motivos y las justificaciones de aquellas personas que han estado involucradas en cualquier forma con las actividades ilegales y criminales. Esto es

\footnotetext{
12 Emsley, Clive, Crime and society in England, 1750-1900, Pearson Education, 2005, p. 158. Para una discusión sobre el robo en la industria lanera, véase Godfrey, Barry \& Cox, David, Policing the Factory: theft, private policing and the law in modern England, 1777-1968, Bloomsbury Academic, 2013.

${ }^{13}$ Godfrey, Barry \& Lawrence, Paul \& Williams, Chris, History and crime, Sage Publications, 2007, p. 130.

${ }^{14}$ Para un análisis detallado de las Leyes Lanera (Worsted Acts) de la década de 1770, el impacto de Fiscalía Lanera (Worsted Inspectorate) y un examen detallado de la usurpación desde el lugar de trabajo entre 1777 y 1968, véase Godfrey, B. \& Cox, D., Policing the Factory, Op. Cit.

${ }^{15}$ Gatrell, Victor, Crime and the law, 1980. Citado en Smithies, E., The black economy, Op. Cit., p. 5.

${ }^{16}$ Smithies, E., The black economy, Op. Cit., p. 112.

17 Testimonio oral obtenido por la autora entre 1996 y 2013.
} 


\section{Revista Historia y Justicia}

ISSN 0719-4153 revista.historiayjusticia.org

No6 - Santiago de Chile, abril 2016, p. 274-294

particularmente relevante para la historia más reciente relativa a la Segunda Guerra Mundial y los años subsecuentes, ya que aún es posible entrevistarnos directamente con aquellos grupos e individuos quienes tienen experiencias de este período, a la luz del hecho que el pequeño delito y las transacciones a pequeña escala del mercado negro (o gris) aumentaron, particularmente durante el consiguiente período de austeridad ${ }^{18}$.

\section{El robo en el período de postguerra}

Durante la Segunda Guerra Mundial el racionamiento y la escasez fueron ampliamente aceptados por la población británica como una consecuencia inevitable del conflicto prolongado. Ellos pudieron haber eludido el sistema en formas menores tendientes a complementar sus dietas o ingresos, considerando esto como "compensaciones por los muchos sacrificios personales hechos a petición del gobierno central" 19, pero generalmente expresaron desaprobación por la actividad criminal a gran escala, o el pandillerismo, condenada como inmoral y antipatriótica por los entrevistados ${ }^{20}$. Fue, de todos modos, el subsecuente período de austeridad y extensión de restricciones, el que aumentó el resentimiento público e intensificó los patrones de comportamiento criminal. En 1949, Silcock planteó que "el robo ha aumentado de manera importante durante los pocos años pasados y continúa creciendo" 21 . Según su estimación, esto fue a causa del "crecimiento en la población del elemento que está dispuesto a comprar a través de canales ilegales sin indagar demasiado acerca de la propiedad de los bienes que le ofrecen en venta"22. Quienes hubieron aceptado las restricciones durante la Guerra se desilusionaron tras la proyección de estas restricciones y hacia 1950 la paciencia pública parecía agotada -la gente "no estaba preparada para tolerar este tipo de camisas de fuerza en tiempos de paz"23. Por lo tanto, el comportamiento previamente considerado inaceptable, el cual había sido censurado, ahora era, de acuerdo con Roodhouse, legitimado a los ojos de la población británica ${ }^{24}$. El testimonio oral ha revelado las ambivalentes actitudes hacia el crimen en el período de escasez cuando el Dudley Herald observó que "la escasez de muchos productos -alimento, combustible, oferta inmobiliaria y de materiales- (...) parece estar obstruyendo la industria y afectando la totalidad de la vida de la comunidad" 25 . En consecuencia, fue crecientemente tentadora la oferta de productos manufacturados localmente a ser distribuidos por la fuerza

\footnotetext{
18 Smithies, E., The black economy, Op. Cit.

19 Roodhouse, Mark, "Popular morality and the Black Market in Britain, 1939-1955", en Trentman, Frank \& Just, Fleming (eds.), Food and conflict in Europe in the age of the Two World Wars, Palgrave McMillan, 2006, p. 244.

${ }_{20} \mathrm{Mr}$. SR Sedgley; Miss VH Wolverhampton.

21 Silcock, H., The increase in crimes, Op. Cit., p. 10.

22 Silcock, H., The increase in crimes, Op. Cit., p. 10.

${ }^{2}$ Smithies, E., The black economy, Op. Cit., p. 111.

24 Roodhouse, M., "Popular Morality", Op. Cit., p. 260. Esto es corroborado por los entrevistados durante la investigación de la autora.

25 Dudley Herald, 18 de enero de 1947, p. 12.
} 


\section{Revista Historia y Justicia}

ISSN 0719-4153 revista.historiayjusticia.org

Nº6 - Santiago de Chile, abril 2016, p. 274-294

laboral. Los entrevistados explican que ellos vieron esta situación como poco menos que una infracción menor de reglas insignificantes, no como un crimen: "En ese momento particular, donde aún había racionamiento y todo el mundo - la mayoría de la gente- hacía lo que podía, y si tenían la posibilidad de conseguir algo más transando y regateando, cualquier cosa que no sea robar" 26 . La autobiografía de John Petty explicaba las actitudes variables hacia las infracciones menores de la ley: "Los años de guerra y la escasez convirtieron a tanta gente honesta en oportunistas y tramposos, e hicieron a muchos otros tolerantes hacia los sospechosos" 27.

Mediante la escucha de entrevistas directas, los estudiantes podrían ser capaces de construir un panorama más detallado de las actitudes hacia este tipo de comportamiento y evaluar cómo dichas actitudes pudieron cambiar. Los entrevistados explicaron cómo obtenían bienes ilícitos y la persistencia de tales actividades, dando cuenta de la tolerancia entre miembros respetables de la comunidad quienes toleraron este comportamiento, excusando a los perpetradores de las autoridades e involucrándose en actividades sospechosas con creciente regularidad. Mr. JB era un funcionario judicial durante este período, pero su opinión tipifica la perspectiva de otros: "Si tú querías alguna cosa, sólo ibas al bar y preguntabas (...) esta es una comunidad unida y nadie podría delatarte, suerte para ti (...) todos hacían un poco de dinero al margen del mercado" 28 . Otros testimonios confirman su punto de vista: "Siempre podías obtener cosas en el mercado negro. Siempre había alguien que conocía a alguien"; "Después de la guerra seguía el racionamiento y la gente efectivamente conseguía los productos (...) la gente que sabía" ${ }^{29}$. Mr. JW describía a su tío con gran cariño y en tono de broma, como:

“(...) Un personaje (...) Él fue uno de aquellos individuos; él tenía un collar de relojes en sus brazos. Trabajaba de día (sic). Vendía de todo. Consiguió un gran patio y tenía de todo (...) Nunca trabajó de manera convencional y una vez volvió con un mono [vivo] (...) Tocaba el piano (...) Vivía de su ingenio (...) Él era un poco turbio" ${ }^{30}$.

La actividad criminal y los intentos de eludir el sistema se volvieron más comunes, de acuerdo a Roodhouse, con la redefinición del término "mercado negro", que permitía "a los entrevistados superar sus inhibiciones hacia los negocios ilícitos y mantener una imagen

\footnotetext{
${ }^{26}$ Mr. MC Sedgley: "At that particular time there was still rationing going on and everybody -most people- got what they could, and if they were able to get a little bit more by wheeling and dealing, anything other than stealing".

${ }^{27}$ Petty, John, Five fags a day: The last year of a scrap picker, Martin Secker and Warburg Ltd., 1956, p. 48. Petty fue, según su propia definición, un "recolector de chatarra" ("scrap picker") que fue despedido a causa de la mala salud, apoyando en las ganancias de la recolección de basura metálica desde los vertederos en Walsall durante la década de 1940 y 1950.

${ }^{28}$ Mr. JB Tipton.

NdE: el inglés de los entrevistados es lengua popular, y se ha procurado mantener ese rasgo en esta traducción, en la medida que no dificulte la comprensión del texto.

${ }^{29}$ Mr. BJ Pensnett; Mr. JC Tipton.

${ }^{30}$ Mr. JW Tipton.
} 


\section{Revista Historia y Justicia}

ISSN 0719-4153 revista.historiayjusticia.org No6 - Santiago de Chile, abril 2016, p. 274-294

no desviada de sí mismos"31. Esto se ve reflejado en los conceptos utilizados por los entrevistados para describir sus acciones personales y de otros. Ellos recordaban que las acciones aparentemente ilegales eran la "norma", mientras sostenían que las actividades no delictivas tenían lugar, hablando de: "tejemanejes" [wheeling and dealing], "hacer cosas al margen" [bits on the side], "servicios" [obliging], "beneficios" [perks], "golpes de suerte" [knocking off, "evasivas" [evasions], "manejos" [fiddles] que eran parte de la estructura habitual de sus vidas cotidianas. Mr. KD apelaba a que "aquello no era un crimen": "realmente, volviendo a los cincuentas, sesentas y setentas (...) cuando tú decías crimen siempre se trataba de pequeños delitos (...) birlar esto y birlar aquello"32. El crimen y los criminales también eran redefinidos según la historia oral indicando la relación conflictiva entre empleadores y empleados en el lugar de trabajo, lo que cambió las percepciones de los comportamientos aceptados durante la postguerra, ya que, con la escasez de trabajo, brindaron a los trabajadores una mayor cuota de poder ${ }^{33}$.

\section{Justificando el comportamiento criminal}

Según el análisis de Todd sobre las jóvenes mujeres en sus lugares de trabajo durante la primera mitad del siglo XX, se concluía que el resentimiento hacia los dueños $y$ administradores de grandes compañías impulsaba el hurto de productos desde el lugar de trabajo, siendo percibido como una actividad legítima ${ }^{34}$. El testimonio oral corrobora esta perspectiva y arroja luces sobre las motivaciones del hurto. La lectura de transcripciones y escucha de entrevistas por parte de los estudiantes podría significar una posibilidad de analizar las actitudes hacia el crimen y el comportamiento criminal. Por ejemplo, al ser consultada sobre el delito en el lugar de trabajo, Mrs. JW sostuvo: “¿Crimen? No, no con maldad"35. Su marido intervino: "No podía ser calificado como un crimen, porque (...) son criminales quienes dan palizas de sangre, quienes obtienen cosas de manera deshonesta (...) ellas sólo estaban sacando beneficios de la basura (...) no era robar si lo tenían de la compañía en que trabajabas". Esto demuestra claramente que, para algunos individuos, la cultura del robo desde el lugar de trabajo significó que aquellos bienes tomados del taller fueran calificados como beneficios complementarios del empleo, particularmente para conductores de vehículos de despacho y trabajadores no calificados, para quienes "los

\footnotetext{
31 Roodhouse, M., "Popular morality”, Op. Cit., p. 256.

32 Mr. KD Pensnett.

33 Los reportajes periódicos en el Dudley Herald entre 1945 y 1970, indican que los empleadores eran, en cierta medida, más proclives a aceptar el pequeño robo, particularmente cuando buscaban retener la mano de obra calificada. Entrevistas de la autora, 1996-2013.

34 Todd, Selina, Young women, work and family en Englando, 1918-1950, Oxford University Press, 2005, p. 163.

35 Mrs. VW Tipton.
} 


\section{Revista Historia y Justicia}

salarios en las fábricas eran terribles si no estabas calificado" 36. Los patrones acostumbrados de comportamiento fueron aceptados como normas sociales y, como Mr. HT, recalcó: “(...) gente que trabajaba en cualquier parte, se llevaba cosas a casa (...) si había algo que querías, podías conseguirlo si sabías cómo" 37 . El marido de una de las entrevistadas estaba en prisión como resultado del robo contra su empleador y, a través del relato del episodio por parte de su esposa, ella sostuvo enfáticamente el sentimiento de derecho de los trabajadores para acceder a los bienes de sus empleadores ${ }^{38}$. Este tipo de hurto fue, en cierta medida, un reflejo de la evaluación que cada empleado hacía del valor de su propio trabajo, el cual frecuentemente consideraba cierto grado de pago en especies, incluso si el propietario no estaba en conocimiento de ello ${ }^{39}$. Mr. KD describió las actitudes entre sus contemporáneos: "Ellos [los empleadores] pueden permitirse el lujo de perderlo, iporque tienen un montón! Yo creo (...) que era parte del trabajo para ser honesto. Era el producto que nosotros hacíamos"40. Los sentimientos de Mr. JB, de Tipton, tuvieron pleno respaldo entre los entrevistados: "Los patrones son ogros, si puedes obtener lo que sea de ellos, está bien" 41.

A pesar de que los beneficios de tales robos fueron a menudo despreciables, como los precios de metales aumentaron en la década de 1950, "todos se volvieron extremadamente conscientes de la bolsa de metales. Había cualquier cantidad de tipos (en las fábricas) quienes habían llegado a considerar la ratería como orden natural de las cosas" 42 . Los entrevistados confirmaron que "nadie (...) [veía] esto como un crimen o algo anormal" 43 . Paradojalmente, en sus memorias, Petty escribe sobre "trabajadores honestos" quienes cargaban por las calles con bolsos llenos de metal obtenido desde su lugar de trabajo ${ }^{44}$. Otros materiales fueron hurtados de forma regular, incluyendo carbón de los barcos de navegación fluvial atracados en los muelles carboníferos de Tipton. Dichas acciones eran consideradas justificables "si era para mantener a los niños abrigados (...) aquello no era un crimen" ${ }^{45}$. La madre de Mrs. KD se vio obligada, a causa de los altos precios de los combustibles y la necesidad de mantener a su marido enfermo, a unirse a los residentes de Pensnett para robar carbón desde la acería local: “cada noche, la gente de por aquí obtenía el carbón que necesitaba, y mamá era una de

\footnotetext{
${ }^{36}$ Mr. JW Tipton. Los elementos destacados por Mr. JW son indicativos de las actitudes de algunos empleados en el área de Tipton durante el período de postguerra y fueron corroborados por otros entrevistados. De todos modos, Mr SR, opinó que su empleador era "un tipo de cuidado".

37 Mr. HT Tipton.

38 Mr. BW Pensnett(fuera de micrófono).

${ }^{39}$ Badger, Samantha, Household consumption. Food and the working-class: The Black Country and Coventry, 1930-1970, Tesis de doctorado, Universidad de Wolverhampton, 2004, p. 136.

40 Mr. KD Pensnett.

${ }^{41} \mathrm{Mr}$. JB Tipton.

42 Petty, J., Five fags, Op. Cit., p. 50.

${ }^{43}$ Mr. FC Tipton; Mr. JW Tipton; Mr. NR Pensnett.

${ }^{44}$ Petty, J., Five fags, Op. Cit., p. 49. Petty, quien se hizo la vida vendiendo chatarra robada, afirmaba que 20 libras de bronce podían venderse por 30 chelines.

45 Mrs. LL Tipton.
} 


\section{Revista Historia y Justicia}

ellas (...) Para evitar los testigos, lo hacían en la oscuridad de la noche (...) y ellos sabían exactamente cuando la seguridad (...) podría andar rondando"46. El carbón que no era necesario para el uso inmediato de las familias, era vendido o cambiado por otros productos $^{47}$. La justificación para el robo se centraba en los problemas de salud o las necesidades de niños y ancianos. Nuevamente, tales ideas sobre la mentalidad de aquellos involucrados en actividades criminales pueden ser utilizadas para desarrollar la comprensión de los motivos y justificaciones para el robo por parte de los estudiantes. También indican que deben ser consideradas cuidadosamente, tanto las percepciones como las clasificaciones del comportamiento criminal, así como el hecho de que la definición del crimen, en términos de una violación de la ley penal, no coincide necesariamente con las definiciones públicas.

\section{Recibiendo bienes robados}

A pesar de que a veces los bienes robados eran para el consumo personal -como en el caso de los trabajadores de la fábrica Tate and Lyle's, quienes, siendo benditos por el rabino, ocultaron pequeñas cantidades de azúcar en el dobladillo de sus pantalones de trabajo ${ }^{48}$, o aquel de los ladrones de la fábrica de salchichas Palethorpe, presenciado por varios entrevistados ${ }^{49}$, otros entrevistados mencionaron la práctica de robar a pedido. Ellos recordaron la capacidad de hacer pedidos de productos específicos, los cuales podían ser debidamente entregados. Algunos trabajadores tenían una lista de espera de clientes. En un vecindario, donde los residentes fueron descritos como "honestos y esforzados", era posible pedir productos específicos y alguien aparecía con ellos ${ }^{50}$. Mr. JW, en sus recuerdos de los talleres Vono, en Tipton, explicaba las maneras en que productos grandes, como camas y sofás, eran robados a pedido desde las fábricas del Black Country. "La gente de Vono solía colocar sus camionetas junto a las rejas y alguien pasaría las cosas por encima". Él recordaba a su madre -"cristiana y de recto comportamiento"- y a una tía -"que iba regularmente a la iglesia"-, quienes "obtuvieron un horno a gas y una cocina de la fábrica Cannon, por encima del muro" 51.

Algunos individuos, quienes habían sido específicamente contratados para desalentar el robo y proteger los intereses de los empleadores, también fueron cómplices de las transacciones ilegales. Cuando Crestwood Estate estaba en construcción "uno de los muchachos era un guardia de seguridad (...) así que básicamente podías obtener cualquier cosa para la casa que quisieras (...) cuando los desechos de acero inoxidable salían, teníamos

\footnotetext{
46 Mr. KD Pensnett.

${ }^{47}$ Mr. SB Pensnett.

48 Barrett, Duncan \& Calvi, Nuala, The sugar girls: Tales of hardship, love and happiness in Tate \& Lile's East End, Harper Collins, 2012, p. 242-243.

${ }^{49}$ Mr. HT Tipton, Mrs. VW Tipton; Mr. MJ Tipton.

50 Mr. SB Pensnett.

51 Mr. JW Tipton.
} 


\section{Revista Historia y Justicia}

ISSN 0719-4153 revista.historiayjusticia.org N6 - Santiago de Chile, abril 2016, p. 274-294

el camino despejado (...) podías tener lo que quisieras cuando ellos estaban construyendo las casas nuevas" 52 . Los beneficios percibidos por algunos de los guardias fueron tales que uno de los guardias de Pelethorpe "terminó con una casa rodante con la que recorrió todo el camino hasta la costa" de Gales, la cual había sido financiada por sobornos para ignorar el crimen organizado dentro de la empresa ${ }^{53}$. Otro hombre de seguridad en la compañía "puso el ojo en los conductores de despachos" así que él podía intimidarlos para ser pagado con parte de las ganancias de las ventas ilícitas. Un conductor despachaba comida a una tienda, pero cuando iba saliendo de la empresa, el guardia le decía: "No le has pagado por tal jamón (...) dame la mitad [de la ganancia]. Todos los chicos estaban coludidos y tenían miedo de decir que no"54. De manera similar, miembros de la policía podían recibir productos robados o impulsar a los trabajadores a torcer las reglas; en los sesentas "los policías de Pensnett estaban tan torcidos como ellos", a veces recibiendo bienes robados y otras alentando pequeños actos delictivos 55 .

\section{Conclusiones de la investigación}

Las entrevistas ponen de relieve una cantidad de temas interesantes relativos a las actitudes sobre el pequeño delito y, en particular, sobre el crimen en el lugar de trabajo, los cuales tendrán el potencial para motivar el interés de quienes estudian la justicia criminal, además de los estudiantes de leyes, sociología e historia. Estas entrevistas revelaron antecedentes para sugerir causas del incremento del crimen en el período de postguerra y corroboraron afirmaciones de que el período de austeridad de postguerra y la acumulación de finales de los cincuentas y sesentas anunciaban una época en la cual las aspiraciones por poseer bienes de consumo duraderos fueron mayores. La publicidad impulsaba las ambiciones y los patrones de mercado incrementaban las oportunidades para la actividad criminal. Esto corrobora los argumentos relativos a que recibir bienes de origen dudoso no imposibilitaba necesariamente a los entrevistados el autodefinirse como "consumidores éticamente correctos" y también confirma la perspectiva de Smithies, de que "el robo al empleador era una característica de la vida industrial británica, a cuenta de las circunstancias económicas generales". Evidentemente, altos salarios y pleno empleo no eran barreras efectivas contra el robo en el lugar de trabajo, como se comprobó durante el período de 1955 a 1970 en que los entrevistados reportaron los mayores incidentes de "redistribución" de bienes y "expropiación" ["workplace appropriation"] ${ }^{56}$.

${ }^{52}$ Mr. SB Pensnett. Crestwood Estate fue un gran proyecto inmobiliario construido en la zona de Pensnett, en Dudley, durante los años sesenta.

${ }^{53}$ Mr. HT Tipton. Palethorpe's fue el mayor procesador de carne y salchichas con fábricas en las Midlands.

${ }^{54}$ Mr. HT Tipton.

${ }_{55} \mathrm{Mr}$. SB Pensnett.

${ }^{56}$ Roodhouse, M., "Popular morality...”, Op. Cit., p. 244; Smithies, E., The Black Economy..., Op. Cit., p. 115; Godfrey, B. \& Lawrence, P. \& Williams, C., History and crime..., Op. Cit., p. 132. 


\section{Revista Historia y Justicia}

ISSN 0719-4153 revista.historiayjusticia.org No6 - Santiago de Chile, abril 2016, p. 274-294

La disponibilidad de bienes y la floreciente sociedad de consumo sirvieron para exacerbar la problemática: ¿Por qué mantenerse en la lista de espera u ordenar nuevos productos de consumo, cuando los mismos pueden ser fácilmente accesibles desde el cargamento de un camión que los despacha y a un precio más conveniente? La manufactura de determinados productos producía en algunos obreros un cierto sentimiento de derecho -estar involucrado en el proceso de producción resultaba en sentimientos de propiedad, al menos en parte. Un entrevistado recordó la actitud de sus compañeros de trabajo: "Era parte del trabajo, así que yo estaba en mi derecho de hacerlo, a pesar de que era contra la ley". Continuando su explicación con algunas referencias a los antagonismos de clase entre empleador y empleado, como una explicación para el robo, planteó: "Recuerdo a un tipo (...) que tenía 'dedos largos ${ }^{57}$ y que se construyó un garaje y todo lo que iba a dar allí era de Round Oak. Él nunca lo vio como un delito. 'Earl of Dudley's tiene un montón, nunca va a extrañarlo' "58. Inevitablemente, conforme las industrias manufactureras abandonaban una cercana relación de paternalismo, la cual se desarrollaba entre algunos patrones y sus obreros, mientras más distante e impersonal un administrador, era percibido como sujeto de menor lealtad, al menos a los ojos de algunos trabajadores ${ }^{59}$. Los entrevistados a menudo explicaron sus acciones, declarando: "no era como en los viejos tiempos, cuando ellos [los patrones] conocían tu nombre; ellos no lo conocían a uno, no tenían idea de quiénes éramos" 60 . Aquellos involucrados en tales actividades distinguían claramente entre el comportamiento aceptable e inaceptable dentro de la comunidad. Falsificar o engañar al empleador, a la compañía o a entidades anónimas era justificado por varios entrevistados como un gaje del oficio, mientras que robar a los vecinos era percibido como un crimen. Los perpetradores de tales actos [robo en el lugar de trabajo] fueron lealmente protegidos y tolerados, incluso incitados, en tanto los crímenes fueran cometidos contra instituciones impersonales o las autoridades. Las actividades criminales eran juzgadas aceptables cuando tendían a complementar el ingreso familiar, para hacer un favor a algún amigo o familiar, o bien eran dirigidas contra los miembros más adinerados de la sociedad, "quienes podían permitirse perderlo" o "podían nunca extrañarlo realmente"61.

El testimonio oral también proporciona algunas explicaciones posibles para el encubrimiento del crimen, las cuales pueden ampliar la comprensión de los estudiantes sobre la complejidad de cuestiones alrededor de nuestra percepción de las actividades "criminales" y las normas sociales. Comportamientos que son definidos oficialmente como ilegales pueden ser, en determinadas circunstancias, redefinidos y considerados como comportamientos aceptables al interior de determinadas comunidades. Primero, los delitos de

\footnotetext{
${ }^{57} \mathrm{NdT}$ : Forma coloquial para referirse a la habilidad de robar.

${ }^{58}$ Mr. KD Pensnett. Round Oak Steelworks fue fundado por Earl of Dudley en 1857 y empleaba durante su apogeo durante los años 1950s-1960s alrededor de 3.000 trabajadores.

${ }^{59} \mathrm{Mr}$. SR Wolverhampton; Mr. NS Sedgley.

${ }^{60} \mathrm{Mr}$. RB Tipton; Mr. DT Sedgley.

${ }^{61}$ Mr. TD Tipton; Mr. FG Sedgley; Mr. TB Sedgley.
} 


\section{Revista Historia y Justicia}

ISSN 0719-4153 revista.historiayjusticia.org

No6 - Santiago de Chile, abril 2016, p. 274-294

esta naturaleza fueron más fácilmente encubiertos por miembros de comunidades obreras unidas y tradicionales. Mr. JB ratificó que su comunidad "seguía teniendo una actitud de mineros (...) pasada de moda, arraigada (...) todos 'sacaban su tajada' [makes a bit on the side] (...) somos muy parroquianos -nos mantenemos juntos-" 62 . En segundo lugar, los elementos inextricablemente unidos de lealtad dentro del vecindario y la posibilidad de intimidación generaban individuos reacios a delatar a algún miembro de su comunidad ${ }^{63}$. Tercero, la práctica de contratar empleados por recomendación, así como toleraba también facilitaba el robo desde el lugar de trabajo, en zonas donde una gran cantidad de trabajadores encontraba empleo a través de parientes y amigos. Familiares, vecinos y amigos eran considerados más abiertos a encubrir el comportamiento ilícito, que los forasteros. En cuarto lugar, tales individuos fueron más proclives a beneficiarse de las actividades ilegales. Robar productos, hacer algún favor a amigos o familiares fue visto como una práctica legítima "hacerles un favor" y cuando la ocasión se presentaba este comportamiento era probablemente recíproco ${ }^{64}$. Era más aceptable robar y vender un producto de este modo, obteniendo sólo un beneficio nominal, que robar para vender a un precio más alto exclusivamente por lucro ${ }^{65}$. Todo lo cual confirma las aseveraciones de Smithies respecto de que en las provincias "las redes locales de relaciones comerciales, políticas y sociales, especialmente (...) [entre] amigos o colegas", vecinos y compañeros de trabajo contribuyeron a modificar las percepciones y definiciones del crimen y del comportamiento criminal ${ }^{66}$.

Fragmentos de testimonios de esta muestra han servido para arrojar algunas luces sobre las complejas motivaciones, explicaciones y justificaciones para el robo desde el lugar de trabajo; ellos dan cuenta de la complejidad del problema y complementan las estadísticas existentes y, en vista de ello, podrían aumentar y mejorar el compromiso de los estudiantes con las fuentes documentales en general, si pueden establecer vínculos claros entre evidencia sustantiva y subjetiva.

\section{El testimonio oral más allá del crimen de clase obrera y el robo en el lugar de trabajo}

A pesar de que la documentación para este artículo se ha enfocado en el robo por parte de miembros de la clase trabajadora y el hurto desde el lugar de trabajo, hay una gran cantidad de elementos susceptibles para el estudio de otras áreas de la actividad ilegal. Claramente los trabajadores de cuello blanco no están exentos para romper la ley. Algunos entrevistados de clase media han hablado de sus propias experiencias de robo desde el lugar de trabajo, desde robar insumos de papelería hasta pequeñas cantidades de

${ }^{62}$ Mr. JB Tipton.

${ }^{63}$ Mrs. BT Tipton; Mrs. IT Sedgley; Mr. DM Pensnett.

${ }^{64}$ Mr. SG Pensnett; Mr. NR Pensnett.

${ }^{65}$ Mr. SB Pensnett; Mr. NS Sedgley; Mrs. MA Tipton; Roodhouse, M., "Popular morality...”, Op. Cit., p. 258.

${ }^{66}$ Smithies, E., The black economy..., Op. Cit., p. 30. 


\section{Revista Historia y Justicia}

dinero de la oficina. Trabajadores de ambas clases sociales utilizaron el tiempo de sus jornadas laborales y los recursos de sus lugares de trabajo para fabricar "foráneos", ya sean muebles, juguetes o bienes metálicos, los cuales más tarde vendieron; costureras y sastres han aprovechado el tiempo de sus jornadas y materiales de trabajo para producir ropa para la venta, así como arquitectos y proyectistas han hecho lo mismo al elaborar los planos para construcciones y ampliaciones ${ }^{67}$.

El testimonio oral puede revelar actitudes hacia el crimen, las cuales podrían ser difíciles de rastrear a través de otras fuentes, por ejemplo en la transición desde el hurtar en una tienda de esquina al hacerlo en un supermercado. Puede ser utilizado para dirigir interrogantes planteadas por Morris, quien en 1989 indicaba que: "Hacia los sesentas los robos desde las tiendas se han convertido en una característica establecida de la venta por departamentos, a pesar de que no se ha sabido de un gran trato entre los mismos ladrones" 68 . Los entrevistados a menudo sienten que el hurto era aceptable si su objetivo era alimentar la familia y excusaban este comportamiento. Mrs. LL, por ejemplo, planteaba que robar comida no podía ser considerado un crimen "porque sus hijos nunca tenían manzanas" y un Juez de Paz explicaba que las tiendas han tenido que soportar alguna responsabilidad por el hurto al "dejarlo fácil para la gente que lo hace. Estaban [los productos] expuestos justo frente a ti, ¿ves?" ${ }^{69}$. Las perspectivas de quienes eran responsables de hacer cumplir las leyes también son de interés; tanto antiguos oficiales de policía, como funcionarios de aduanas, magistrados y jueces locales, permitirían obtener enfoques sobre la multiplicidad de actitudes sobre el crimen y el comportamiento criminal.

Las entrevistas pueden ser útiles para complementar datos estadísticos sobre las formas de eludir el racionamiento y las faltas relacionadas con el mercado negro porque "debido al problema de las cifras oscuras del crimen no registrado (...) [las cifras de represión] son un reflejo de la actividad represiva más que de la extensión de la actividad ilegal"70. El testimonio oral puede ayudar a los estudiantes en una comprensión más profunda de los motivos de los ciudadanos previamente respetuosos de las leyes, que comenzaron sus correrías en el mercado negro, el trueque y el pequeño fraude. Sus justificaciones para aquello que sólo puede ser descrito como un comportamiento criminal son interesantes; desde quienes argumentaron que "no puede ser calificado como crimen" a la explicación de Mrs. IT, según la cual: "Bueno, todo el mundo estaba cansado de andar sin nada, así que hicimos lo que pudimos para ayudarnos entre nosotros"71.

${ }^{67}$ Mr. RA Pensnett; Mr. JC Tipton; Ghent, B., "Lead cowboys made in a Tipton foundry", The Black Country Bugle, 15 de diciembre de 2011, p. 15.

${ }^{68}$ Morris, T., Crime and criminal justice, Op. Cit., p. 98.

${ }^{69}$ Mrs. LL Tipton; Mr. ¿?C Tipton.

${ }^{70}$ Roodhouse, M., "Popular morality...”, Op. Cit., p. 249.

${ }^{71}$ Miss YB Pensnett; Mrs. IT Sedgley. 


\section{Revista Historia y Justicia}

ISSN 0719-4153 revista.historiayjusticia.org N6 - Santiago de Chile, abril 2016, p. 274-294

Las preguntas relativas al abastecimiento de carne, por ejemplo, han sugerido evidencia sustantiva para proponer que el tradicional, aunque ilegal, método de complementar el suministro de alimentos e ingresos mediante la caza furtiva continuó durante la posguerra, más de lo que se ha supuesto. Como confirman los entrevistados: "había un montón de conejos; nunca sabías de dónde venían" y "si querías carne barata, era mejor no averiguar demasiado sobre su origen" 72 . Los entrevistados citados en la tesis de Barger confirman la continuidad de esta práctica, y Mr. SB recordaba cazar furtivamente con sus amigos a menudo en el terreno del Conde de Dudley, en cualquier sitio desde Pensnett a Kinver ${ }^{73}$.

Evidentemente, la metodología de la historia oral tiene el potencial de ser utilizada como un recurso pedagógico para exponer eventos, miradas e incidentes referidos al crimen y la actividad criminal, que de otra manera podrían permanecer ocultos. Tal metodología puede ser utilizada junto con fuentes tradicionales y cifras estadísticas para profundizar el estudio de la historia del crimen y complementar las fuentes existentes.

\section{Historia oral: trampas y críticas}

Las entrevistas orales son usadas frecuentemente para contrapesar los estudios sociales, la evidencia estadística y los informes oficiales, y pueden suministrar tanto un complemento como un desafío a los presupuestos y teorías existentes. No son una fuente nueva para los historiadores o los científicos sociales; pero han recibido críticas en el pasado por ser consideradas inherentemente no fidedignas. Aun cuando podría ser inapropiado desarrollar una prolongada defensa del uso de la historia oral aquí, es necesario considerar brevemente sus críticas y dificultades metodológicas ${ }^{74}$. No obstante, las entrevistas orales son evidencia y, como Elton argumentó en 1967, es importante analizar todo el material relevante -“los vestigios físicos de los episodios a estudiar" - y, por supuesto, no puede haber dudas que la gente que comparte sus memorias y experiencias en la forma de la historia oral forma parte de los "vestigios físicos" de Elton ${ }^{75}$. Los primeros exponentes del uso de entrevistas orales en la investigación histórica y social enfrentaron acusaciones relativas a cuestiones fundamentales, como la inexactitud de la memoria, la infalibilidad de los hechos y la subjetividad. Tales cuestionamientos han sido ampliamente abordados por Paul Thompson, uno de los pioneros de la historia oral. En su opinión, existe una diferencia cualitativa mínima entre el testimonio oral y otras fuentes tradicionales; la solución planteada por Thompson fue que las entrevistas deberían ser sujetas a la crítica de fuentes acostumbrada,

\footnotetext{
72 Mr. JB Tipton; Mr. MJ Tipton; Mrs. OH Sedgley.

73 Badger, S., "Household consumption, food and the working-class: the Black Country and Coventry, 1930-1970", PhD Thesis, University of Wolverhampton, 2004, p.136-140; Mr. SB Pensnett.

74 Véase Dunaway, David, "Method and theory in the oral biography", Oral History, n²0, 1992, p. 40-44.

75 Elton, Geoffrey, The study and practice of History, Fontana Press, 1967, p. 87-113. En este capítulo, Elton discute "Las fuentes" y "Evidencia y crítica".
} 


\section{Revista Historia y Justicia}

ISSN 0719-4153 revista.historiayjusticia.org No6 - Santiago de Chile, abril 2016, p. 274-294

desde el enfoque de que al igual "que todas [las fuentes] son falibles y sesgadas"76. El testimonio oral, donde sea posible de ser desarrollado, apoyará "una construcción más realista y justa del pasado, un desafío al discurso establecido" 77 y de esta manera ampliará las bases para la enseñanza de la historia del crimen.

La problemática cuestión de la falibilidad de la memoria es abordada por Thompson desde el punto de vista de que "toda información es retrospectiva y el único problema extra que tenemos con las entrevistas históricas es que el espacio de tiempo [entre el hecho histórico y el testimonio] es más largo"78. No obstante, es necesario que consideremos las críticas correspondientes a la selectividad de la memoria, la posibilidad de olvidos sobre un período prolongado de tiempo y el deterioro de la memoria. Lummis ha tratado con estos problemas con algún detalle, así que basta con decir que muchos individuos tienen la capacidad de recordar el pasado con suficiente claridad y las funciones de la memoria a largo plazo durante el envejecimiento a menudo son conservadas, mientras las funciones de la memoria a corto plazo son las que se dañan ${ }^{79}$. Si es que, siguiendo a Lummis, "el tiempo y la distancia desde una situación debilita el poder de reorganizar [los recuerdos]", los entrevistados pueden tornarse más sinceros y seguros de sí mismos con la edad ${ }^{80}$.

También es preciso plantear que la inmediatez de las entrevistas, a diferencia de las memorias y las cartas, no facilita la introspección prolongada ni proporcionan tiempo para fabricar o aumentar excesivamente la narrativa de los individuos respecto de sus propias experiencias. Pero la precaución sigue siendo necesaria, porque como ha demostrado Summerfield, es inevitable que los hechos del pasado sean revalorados a la luz del conocimiento subsecuente y la experiencia y memorias no pueden ser independientes de las influencias culturales cuando "las construcciones discursivas del pasado (...) cubren (...) [la memoria] de consideraciones e interpretaciones posteriores"81. Así como los individuos, cuando recuerdan incidentes pasados, automáticamente les otorgan significados mediante una interpretación de la cultura dominante, las dificultades de utilizar las narrativas orales como fuente son similares al uso de reportes escritos de vidas individuales porque, como Schlesinger ha indicado, "al final la autobiografía es una interrogación del pasado por el presente" 82 .

\footnotetext{
76 Thompson, Paul, The voice of the past, Op. Cit., p. 152. En el capítulo 4, el autor propone una discusión detallada de las dificultades y beneficios de fuentes específicas.

77 Thompson, P., The voice of the past, Op. Cit., p. 7.

78 Thompson, P., "Problems of method in oral history", Oral History, vol. 1, n4, 1971, p. 4.

${ }^{79}$ Lummis, Trevor, Listening to history, Hutchinson, 1987, p. 117.

${ }^{80}$ Lummis, T., Listening to history, Op. Cit., p. 117.

81 Summerfield, Penny, "Culture and composure: Creating narratives of the gendered self in oral history interviews", Cultural and Social Theory, n¹, 2004, p. 66.

82 Schlesinger, Arthur, A life in the Twenieth Century: Innocent beginnings, 1917-1950, Houghton Mifflin, 2000 , p. xiii.
} 


\section{Revista Historia y Justicia}

ISSN 0719-4153 revista.historiayjusticia.org

No6 - Santiago de Chile, abril 2016, p. 274-294

En consecuencia, en vista del sesgo inherente a cualquier fuente, la consistencia y exactitud de la memoria puede ser verificada de la misma manera que otras fuentes, cuestionando “¿cómo 'encaja' en lo que sabemos del tema?" 83. La triangulación expresada en la comprobación de los hechos con otras fuentes (incluyendo periódicos y otros entrevistados) y la aclaración de datos y fechas con el entrevistado, puede ayudar a confirmar y validar el testimonio de los informantes. Con tales precauciones, las críticas se pueden abordar.

\section{5. ¿Cómo incorporar las entrevistas orales a la enseñanza de la historia del crimen?}

Habiendo discutido las implicancias de la evidencia oral, es necesario considerar dónde y cómo los estudiantes pueden acceder al testimonio oral. En este momento es importante notar que este artículo no está sugiriendo que los estudiantes participen en entrevistas directas con la hermandad criminal, como su seguridad personal es de suma importancia, véase la discusión a continuación ${ }^{84}$. De todos modos, los estudiantes tienen acceso a dos fuentes potenciales, cada una de las cuales proporcionará enfoques originales para su investigación: entrevistas conducidas por estudiantes y entrevistas disponibles en archivos de historia oral.

\section{Conduciendo entrevistas - entrenamiento en historia oral ${ }^{85}$}

El esfuerzo de capacitar a los estudiantes para conducir sus propias entrevistas es una oportunidad ideal para la cooperación transdisciplinaria entre diferentes escuelas, departamentos y facultades. Existen buenos canales de información disponibles para conocer la oferta de tales cursos. La primera parada obligada para quienes desean incorporar el testimonio oral en la enseñanza del derecho y la justicia criminal podría ser el sitio web de Oral History Society, que alberga información exhaustiva de procedimientos, consejos, recursos y lineamientos éticos para el desarrollo de entrevistas, para la transcripción y el consiguiente almacenamiento de información ${ }^{86}$. La Sociedad ofrece jornadas de capacitación y seminarios a nivel local y nacional, para entrevistadores con y sin experiencia; entrega consejos técnicos sobre cada aspecto de la historia oral; y edita el Oral History Journal-cuyo

\footnotetext{
83 Gluck, Sherna, “What's so special about women?: Women's oral history”, en Dunanway, David \& Baum, Willa (eds.), Oral bistory: An interdisciplinary anthology, Sage Publications, 1996, p. 218.

${ }^{84} \mathrm{La}$ información utilizada para este caso de estudio fue el resultado de mi propia investigación en comunidades de clase obrera y no una solicitud de trabajo de investigación como requerimiento de programa de clases.

${ }^{85} \mathrm{La}$ seguridad de los estudiantes es de suma importancia y los lineamientos éticos de ciertas universidades deben ser cumplidas estrictamente. Esto implica la inscripción en las políticas de evaluación de riesgos, las directrices de salud y seguridad y, formularios de propuesta para este tipo de proyectos sometidos a un riguroso escrutinio antes de que sean autorizados por los Comités de Ética.

86 Véase Oral History Society, http://www.ohs.org.uk/.
} 


\section{Revista Historia y Justicia}

ISSN 0719-4153 revista.historiayjusticia.org

No6 - Santiago de Chile, abril 2016, p. 274-294

primer número fue publicado por la Oral History Society en 1971-, que contiene tendencias actuales en la historia oral, consejos y noticias.

Nuevas directrices y consejos están disponibles también en la sección "Making History" del sitio web del Institute of Historical Research, que contiene artículos, recursos, información relativa a los proyectos de investigación en desarrollo y sugerencias para lecturas complementarias ${ }^{87}$. Existen otras innumerables publicaciones académicas sobre historia oral, nacionales e internacionales, una pequeña selección de las cuales incluye: The Oral History Review, una revista para la "teoría y práctica de la historia oral" publicada en Estados Unidos $^{88}$; Words and Silences, que es la revista electrónica de International History Association ${ }^{89} ; \mathrm{y}$ Oral Tradition Journal0. Estas publicaciones entregan invaluables consejos, pautas y sugerencias para nuevas metodologías de investigación y nuevas ideas de todo el mundo.

Para los principiantes en este campo hay dificultades a superar, incluyendo la supervisión de estudiantes en el campo, pero estas dificultades no son generalmente insuperables. Los estudiantes requieren inevitablemente la formación y la práctica para llevar a cabo este tipo de investigación, pero las técnicas adquiridas son habilidades transferibles que les serán útiles en su formación y futuras carreras. Necesitarán orientación sobre cómo alcanzar habilidades en la formulación y dirección de entrevistas, qué tipo de técnicas para entrevistas usar y, aún más importante, la madurez y el tacto para lidiar con cuestiones sensibles y emotivas derivadas del proceso de recordar eventos específicos y, más importante, su impacto sobre los entrevistados; pero hay abundantes consejos al respecto, tanto en forma impresa, como online.

Por supuesto, los lineamientos éticos de la entrevista oral deben ser de la mayor consideración a fin de que el entrevistado tenga la mayor confianza de que el marco legal y ético, bajo el cual la entrevista tiene lugar, le suministrará la protección de sus intereses. La manera en que las entrevistas son presentadas, conducidas, registradas, almacenadas y utilizadas es de vital importancia, particularmente en lo relativo a la confidencialidad y a las leyes de derecho de autor. Una vez más, se puede acceder a consejos y directrices claras sobre tales factores en el sitio web de Oral History Society ${ }^{91}$. Nuevamente, es vital reiterar que la seguridad de los estudiantes es de suma importancia y no se sugiere que puedan ser expuestos a situaciones difíciles o peligrosas (véase la nota 81). Los estudiantes de licenciatura necesitarían aprobación ética para sus proyectos de investigación y son usualmente entregados sólo para entrevistar gente conocida de ellos o del cuerpo académico.

\footnotetext{
87 Véase http://www.history.ac.uk/makinghistory/. Véase, por ejemplo, Smith, Graham, "The making of oral history", 2008. Disponible en http://www.history.ac.uk/makinghistory/resources/articles/oral_history.html.

88 The Oral History Review, http://www.oralhistory.org/publications/oral-history-review/.

89 Word and Silences, http://iohanet.org/journal/index.html.

90 Oral Tradition Journal, http://journal.oraltradition.org/.

${ }^{91}$ Lineamientos éticos de Oral History Society, http://www.ohs.org.uk/ethics.php.
} 


\section{Revista Historia y Justicia}

ISSN 0719-4153 revista.historiayjusticia.org No6 - Santiago de Chile, abril 2016, p. 274-294

El entrenamiento técnico también es una parte vital del proceso. Los estudiantes también tendrán que aprender cómo utilizar los dispositivos de grabación correctamente a fin de que el entrevistado sea claramente audible, y necesitan practicar la descarga y consiguiente transcripción de entrevistas. Con el uso de dispositivos de grabación audiovisual hay incluso más dificultades relativas a los requisitos técnicos adicionales, tales como la ambientación, la claridad y la calidad de la cinta. De nuevo, cierta información concerniente a temas específicos de la historia oral, incluyendo los múltiples desafíos técnicos, está disponible en libros y revistas. Los trabajos pioneros de Thompson, así como las guías teóricas y prácticas como Oral History Theory y Doing Oral History: A practical guide $e^{92}$, están dirigidas a profesores y estudiantes. La segunda edición de Recording Oral History; A guide for the bumanities and social sciences, de Valerie Yow, cubre la gama completa de los tecnicismos y las ventajas de la historia oral; la autora incluye nuevo material sobre el uso de Internet, discusiones sobre la compleja relación entre historia oral y memoria, así como consideraciones para interpretar la información entregada por los entrevistados ${ }^{93}$. "Blueprint for teaching oral history", de K'Meyer, entrega una visión de conjunto de las estrategias empleadas en las universidades estadounidenses por colegas para la enseñanza de la historia oral, e incorpora sugerencias para planificar clases ${ }^{94}$. Más recientemente, el trabajo de Sloan sobre pedagogía oral considera las experiencias de los narradores del testimonio oral. Con estudiantes involucrados como entrevistadores y narradores, examina las experiencias de los participantes en las entrevistas y discute sobre "las diferencias de poder que son frecuentemente inherentes al trabajo en el campo de la historia oral" y proporciona enfoques sobre el sentir y pensar de los entrevistados ${ }^{95}$. Esto es particularmente relevante para incitar la reflexión de los estudiantes, una vez que las entrevistas han sido conducidas y transcritas, los pasos finales corresponden a la interpretación y análisis de los testimonios y sus contribuciones a la comprensión de los estudiantes deben ser evaluadas.

\section{Archivos de historia oral}

Claramente el desarrollo de entrevistas, su consiguiente transcripción y análisis, puede ser un proceso extremadamente prolongado y, en consecuencia, una sugerencia alternativa podría ser el uso de los archivos de historia oral existentes. Las entrevistas pueden ser consultadas y estudiadas desde una amplia variedad de organizaciones, archivos y sitios electrónicos, algunos de los cuales tienes mejor accesibilidad que otros. Para aquellos estudiantes que deseen acceder a información y memorias de crimen ello puede ser

92 Thompson, P., The voice of the past, Op. Cit.; Abrams, Lynn, Oral History Theory, Routledge, 2010; Ritchie, Donald A., Doing oral history: A practical guide, Oxford University Press, 2003 (2 $\left.2^{\mathrm{a}} \mathrm{ed}.\right)$.

${ }^{93}$ Yow, Valerie R., Recording oral history: A guide for the bumanities and social sciences, Altamera Press, 2005 ( $2^{\mathrm{a}} \mathrm{ed}$.).

${ }^{94}$ K'Meyer, Tracey E., " 'It's not just common sense': A blueprint for teaching oral history”, Oral History Review, vol. $25, n^{\circ} 1,1998$, p. 35-56.

${ }^{95}$ Sloan, Stephen, "On the other foot: Oral history students as narrators", The Oral History Review, vol. 39, n², 2012, p. 289-311. 


\section{Revista Historia y Justicia}

ISSN 0719-4153 revista.historiayjusticia.org

No6 - Santiago de Chile, abril 2016, p. 274-294

problemático algunas veces, dependiendo del modo en que las entrevistas disponibles en cada acervo hayan sido catalogadas. La amplia categorización inicial en muchos de los sitios web y archivos no necesariamente incorporan la categoría de crimen, pero una búsqueda más profunda puede conducir a los estudiantes a áreas de interés. Por ejemplo, la sección de historia oral de la British Library contiene una extensa recopilación de antiguas entrevistas de historia oral y "contiene colecciones concernientes a un amplio rango de áreas temáticas" 96. No existe alguna sección categorizada como crimen en los encabezados de búsqueda inicial, pero cuando la palabra "crimen" es ingresada en el catálogo Sound and Moving Image, pueden ser consultadas 3.881 referencias; inevitablemente el proceso de filtrar archivos requiere esfuerzo, pero puede ser gratificante para el investigador diligente y ciertamente podría ser menos engorroso que desarrollar un proyecto de historia oral independiente ${ }^{97}$. Un número de entrevistas potencialmente relevantes, cada una de las cuales tiene un índice de contenido transcrito y números de las pistas de audio relevantes, copias de las cuales pueden ser obtenidas, sujetas a derechos de autor. Varias universidades, grupos comunitarios, bibliotecas públicas, concejos municipales, autoridades locales e individuos tienen sus propios archivos de historia oral y sus sitios web a menudo albergan un motor de búsqueda a través del cual puede obtenerse cierta información inicial. Por ejemplo, el Grupo de Historia Oral de Ambleside, el colectivo Voces de Somerset, o el Centro de Historia Oral Escocesa dependiente de la Universidad de Strathclyde. Una búsqueda básica en el catálogo de la Asociación de Historia Oral de East Midlands utilizando la palabra clave "crimen", como criterio de búsqueda, permite acceder a numerosas entrevistas y proyectos, algunos de los cuales están disponibles online y pueden ser utilizados. Una búsqueda avanzada permite el acceso a fuentes adicionales ${ }^{98}$. Pero un punto de entrada para la investigación podría ser el website de Archivos Nacionales, el que proporciona listas generales de entrevistas de historia oral y transcripciones relativas a una amplia variedad de temas, cuyo uso eficiente de búsquedas avanzadas y palabras clave permitirá acceder a fuentes potenciales adicionales, desde colecciones privadas hasta aquellas almacenadas en los archivos y oficinas de registro ${ }^{99}$. Además de archivos y colecciones de testimonios orales en las Islas Británicas, existen numerosos archivos internacionales que también están disponibles para investigaciones.

Buscar memorias de épocas de guerra y/o racionamiento en archivos de historia oral frecuentemente resultará revelador de las formas de eludir el sistema de racionamiento, la

${ }_{96}$ The British Library, http://www.bl.ok/oralhistory.

97 The British Library, catálogo Sound and Moving Images, disponible en http://cadensa.bl.uk/uhtbin/cgisirsi/?ps=aK5QXxkudz/WORKS-FILE/76060095/123. Consultado el 4 de noviembre de 2013.

${ }^{98}$ East Midlands Oral History Association Catalogue, disponible en http://www.le.ac.uk/emoha/catalogue/subject.html. Consultado el 4 de noviembre de 2013. La variedad de material disponible incluye desde la entrevista al alcaide retirado de una cárcel hasta otras efectuadas a ex miembros de la fuerza policial.

99 The National Archives, en

http:/ /www.nationalarchives.gov.uk/a2a/results.aspx?tab=2\&Page $=1 \&$ ContainAllWords $={ }_{\text {oral }}+$ interviews. 


\section{Revista Historia y Justicia}

ISSN 0719-4153 revista.historiayjusticia.org

No6 - Santiago de Chile, abril 2016, p. 274-294

caza furtiva y otros aspectos relativos a las actitudes hacia el comportamiento criminal desde los sistemas de trueque hasta el robo desde el lugar de trabajo. Sólo un ejemplo de Brighton and Hove podría permitir a los estudiantes el acceso inmediato a registros audiovisuales que detallen las actitudes hacia las transacciones en el mercado negro ${ }^{100}$. Con los avances tecnológicos, los blogs permiten a las personas registrar sus memorias de manera anónima, un blog que fue posteriormente impreso en el Caernarfon Herald reveló ambigüedades con respecto al mercado negro: "Mis abuelos eran gente honesta, que iba a la iglesia, quienes no podrían haber soñado hacer nada con artimañas y astucias (...) Sin embargo, ellos y mucha gente como ellos estaban listos para participar en el mercado negro"101.

Inevitablemente, dada la naturaleza de la actividad criminal y los recuerdos relativos al crimen, investigar este tema mediante los archivos de historia oral puede requerir mucho tiempo y resultar complejo. De todos modos, escuchar un número limitado de registros, o leer algunas transcripciones, complementará las fuentes existentes disponibles para los estudiantes y los ayudará a enfocar sus estudias hacia nuevas perspectivas y los comprometerá con la investigación de nuevas maneras. Es particularmente fructífero para la enseñanza cuando los proyectos colaborativos con otros departamentos son poco prácticos o cuando las restricciones del tiempo imposibilitan a los estudiantes el conducir entrevistas por sí mismos. Quizás, para su primera aproximación al uso del testimonio oral, los estudiantes podrían ser provistos de un número limitado de registros o transcripciones, las cuales puedan ser evaluadas según su utilidad para la comprensión del crimen y el comportamiento criminal. Todos los usos posibles son considerables.

\section{Conclusión}

Tal como se dijo al principio, este artículo no es una guía para el uso de la historia oral en el campo del derecho y la justicia criminal, es más bien una sugerencia -de una historiadora y practicante de la historia oral- de que escuchar la voz de los involucrados, o leer la transcripción de sus entrevistas, puede ser una experiencia (in)formativa para los estudiantes. De manera inevitable, para aquellos practicantes de la historia oral que no están familiarizados con la incorporación del testimonio oral en sus metodologías de enseñanza, o aquellos con experiencia limitada en el campo, esta puede ser una empresa de enormes proporciones. De todos modos, tales dificultades no son insalvables, ya que existe un amplio margen para la cooperación transdisciplinaria y las ventajas para los estudiantes que han accedido a registros de primera fuente de los individuos que se han visto envueltos, de alguna manera, en las actividades criminales son infinitas. Potencialmente, los estudiantes pueden tener acceso a la experiencia de los perpetradores, las víctimas, los testigos y aquellos

100 Brighton \& Hove, disponible en http://www.brighton-hoverpml.org.uk/discoveryandlearning/heritagelearning/resources/Pages/BrightonduringWorldWarIIMedia.aspx.

101 Caernarfon Herald, disponible en http://blogs.caernarfonherald.co.uk/hywelroberts/2011/09/no-64-the-blackmarket-in-ww2.html. Consultado el 29 de septiembre de 2013. 


\section{Revista Historia y Justicia}

ISSN 0719-4153 revista.historiayjusticia.org N6 - Santiago de Chile, abril 2016, p. 274-294

involucrados en el sistema judicial. Esta metodología puede ser utilizada como un apoyo para la comprensión del crimen, para explicar las percepciones sobre el crimen y las actividades criminales en variedad de situaciones, y para explorar las reacciones contemporáneas sobre el crimen entre los miembros de una comunidad.

El testimonio oral tiene el potencial de ampliar la evidencia cualitativa y cuantitativa, ayudar en la interpretación de los documentos, las estadísticas y las legitimidades del crimen. La historia oral ha sido incorporada exitosamente en los programas curriculares de la Maestría en Criminología y Derecho Penal de la Keele University ${ }^{102}$ y en numerosas instituciones de enseñanza superior de Estados Unidos y Australia103. Quizás, entonces, algunas de estas técnicas pueden ser utilizadas para que la experiencia de los estudiantes sea ampliada a través del acceso a entrevistas orales. Puede ayudar a iluminar las cuestiones morales y éticas relativas a la actividad criminal, proporcionando al menos explicaciones parciales de porqué son cometidos determinados crímenes, las circunstancias bajo las cuales determinados tipos de comportamiento criminal han sido toleradas y porqué los perpetradores pueden ser encubiertos por las relaciones sociales del vecindario. Las memorias de los entrevistados pueden, de alguna manera, ayudar a los estudiantes a interpretar la evidencia, contextualizar los patrones de comportamiento, las relaciones de poder, las percepciones de la ley y, de manera enfática, aproximarse a las fuentes tradicionales desde nuevas perspectivas, así como las fuentes tradicionales se incrementan con las experiencias personales.

Traducido por

Ignacio AYALA CORDERO

102 Ver http://www.keeles.ac.uk/media/keeleuniversity/fachumsocsci/sclsocrim/criminology/Glossy\%20leafletMA\%20CCJ-April\%202013\%20update.pdf.

103 Por ejemplo, Kunselman, J. C. \& Johnson, K. A., "Using the case study method to facilitate learning", College Teaching, vol. 52, $\mathrm{n}^{\circ} 3, \quad 2004$, p. 87-92; http://www.princeton.edu/.../content/data/oral_history/; http://tarltonguides.law.utexas.edu/content.php?pid=101468\&sid=763468; Israel, Mark, "Teaching criminology through interview-based assignments", Legal and Educational Review, n¹41, 1997; Departamento de Estudios Legales, Flinders University of South Australia, disponible en http://www.ler.edu.au/Vol\%208_2/Israel.pdf. 